\title{
De Sanctis nella tradizione critica catalana ${ }^{1}$
}

\author{
Gabriella Gavagnin
}

Universitat de Barcelona

gavagnincapoggiani@ub.edu

\begin{abstract}
Malgrado siano pochissime e parziali le traduzioni in catalano di testi di Francesco De Sanctis, nel corso del Novecento le sue opere hanno avuto una notevole diffusione fra scrittori e critici catalani, di cui sono prova l'ampio repertorio di esemplari conservati in biblioteche private e pubbliche, gli interventi critici apparsi su pubblicazioni periodiche e le precise influenze riscontrabili nella produzione saggistica catalana. Il presente studio traccia le linee principali della fortuna desanctisiana in Catalogna, soffermandosi soprattutto sul ruolo svolto da Carles Riba, Josep M. Capdevila e Josep Pla.

Parole chiave: Francesco De Sanctis; critica letteraria; ricezione catalana della cultura italiana.
\end{abstract}

\section{Abstract}

Despite the fact that there are very few and partial translations into Catalan of the texts by Franceso De Sanctis, during the twentieth century his works were widely circulated by Catalan writers and critics. Evidence of this is the ample repertoire of book holdings kept in private and public libraries, their critical presence in periodicals and the precise influence these had in the Catalan essay production. The present study draws the main lines of the Desanctisian production in Catalonia, focusing on the role played by Carles Riba, Josep M. Capdevila and Josep Pla.

Keywords: De Sanctis; literary criticism; Catalan reception of Italian culture.

Il libro che Josep Pla, ormai ultraottuagenario, dedicò all'Italia prende avvio da un'osservazione sulla fortuna, a suo giudizio diametralmente opposta, che in Catalogna hanno avuto Machiavelli e De Sanctis. Sicché, se Machiavelli non ha bisogno di presentazione alcuna e gode, anzi, di una lunga schiera di

1. Questo lavoro è stato svolto nel quadro del Progetto di Ricerca FFI2008-02987: Intercambios entre sistemas literarios: mediación y mediadores desde la literatura catalana (s. XX). 
fautori e di detrattori, «¿quantes persones d'aquest país — si chiede $\mathrm{Pla}$ coneixen la «Storia della letteratura italiana» de Francesco De Sanctis, que és un dels llibres apareguts a Europa el segle passat en el qual es troba el meravellós paper sobre el secretari de Florència» che Pla si accinge appunto a tradurre nel suo libro? ${ }^{2}$ La risposta fa appello all'esperienza personale, notoriamente segnata da un bagaglio nutritissimo di cose e uomini visti: «N'he conegudes poquíssimes, i que el conegui bé, cap». Simile considerazione sembra azzerare in partenza ogni possibile ipotesi di una ricezione catalana di De Sanctis, ridotta a esigue testimonianze disperse di una conoscenza peraltro superficiale. A distanza di più di un secolo, la Storia di De Sanctis continua ad essere ignota ai catalani, afferma Pla, che coglie l'occasione per riscattarla dall'oblio, affidandole il compito di fornire al lettore, con l'«admirable notícia» di Machiavelli in essa contenuta, la porta d'ingresso nella galleria di personaggi storici, "enormes personalitats», ${ }^{3}$ di cui si è nutrita la cultura italiana e su cui si incardina la prima parte del libro Itàlia i el Mediterrani.

Sul De Sanctis di Pla, che traduce nello stesso volume anche il capitolo su Aretino torneremo poi. Quel che importa ora è capire fino a che punto questa affermazione tanto sbrigativa quanto scoraggiante sulla mancata fortuna desanctisiana sia fondata e ponderata, o quanto piuttosto non offra una visione fin troppo, e forse anche tendenziosamente, ridimensionata. Certo, se si parte da un censimento delle traduzioni, il bilancio è quanto mai magro. L'unico De Sanctis catalano fino al libro di Pla risale al 1921, quando Josep Maria Capdevila, nel contesto del centenario dantesco, tradusse i capitoli della Storia dedicati al Trecento e alla Divina Commedia in un volume edito da Editorial Catalana. ${ }^{4}$ Non che il panorama fosse molto più confortante sul fronte delle traduzioni spagnole, in cui si pubblicarono solo due antologie, ambedue opera dello stesso traduttore, Armando Álvaro Vasseur, una nel 1917, Prohombres de Italia, e un'altra di saggi danteschi nel $1919 .{ }^{5}$ Così, mentre la Storia e alcune altre opere desanctisiane videro la luce in versioni argentine del dopoguerra, in Spagna, stando ai dati di cui ho notizia, non si pubblica altro, salvo l'aggiunta dei capitoli tradotti da Pla nel volume citato ${ }^{6}$ e, ancora più di recente, nel 1988, del saggio su Guicciardini recuperato in appendice a una tradu-

2. J. PLA, Itàlia i el Mediterrani, Barcelona: Destino, 1988 [1980ํ], p. 13.

3. Ibid., p. 9.

4. Cfr. F. De Sanctis, Crítica de la Divina Comèdia, Barcelona: Editorial Catalana, 1921. Un estratto del libro fu pubblicato sul periodico La Revista, VII, n. 138, 16 giugno 1921, p. 183-184: «Del Purgatori (Fragment del llibre Crítica de la Divina Comèdia)».

5. Prohombres de Italia: Manzoni, Guicciardini, Mazzini, Cantú, Madrid: Ed. América, 1917 [basata su Scritti varii inediti o rari di F. De Sanctis, a cura di B. Croce]; En torno a la Divina Comedia: ensayos críticos, Madrid: Ed. América, 1919 [basata su Quattro saggi danteschi, a cura di F. Moroncini]. Ricavo questi dati dal Catalogo on-line del Progetto Boscán: Catálogo de las traducciones españolas de obras italianas (hasta 1939). http://www.ub.edu/ boscan. [Consultato il 21/01/2011].

6. Alcuni stralci del capitolo su Machiavelli erano stati tradotti da Pla nell'articolo «El secretari de Florència: Machiavelli» apparso su Destino il 25 aprile 1970, ora ristampato nel volume Per acabar, Barcelona: Destino, 1992. 
zione in spagnolo dei Ricordi. ${ }^{7}$ Insomma, la Storia, di cui Pla constatava la latitanza nella cultura del paese, non era stata e non è stata a tutt'oggi mai tradotta né in catalano né in spagnolo.

Tuttavia, la negazione planiana della conoscenza del libro per eccellenza di De Sanctis nella cultura catalana non può essere confermata o smentita solo sulla base delle traduzioni, posto che la scarsezza o rarità di traduzioni era abbastanza generalizzata in materia di saggistica letteraria, almeno in ambito catalano, malgrado l'evidente influenza della critica straniera su autori catalani. ${ }^{8}$ Anzi, potremmo capovolgere la valutazione appena fatta, perché il libro di De Sanctis tradotto da Capdevila diventa in tal senso un'eccezione tanto esemplare quanto significativa del protagonismo che riuscì a raggiungere il critico italiano. Nondimeno, va tenuto conto che, in Catalogna, in un'ampissima cerchia di letterati, scrittori, intellettuali e artisti, nonché di politici, notabili e professionisti, sono circolati da secoli i libri in italiano, a prova di una ricezione diretta di tutta la cultura artistica, letteraria, tecnica, scientifica e filosofica che dall'Italia potesse arrivare. È in questa densa tradizione che bisogna cercare per accorgersi di quanto sia profonda e diffusa la conoscenza di De Sanctis, del De Sanctis della Storia e di tutte le altre sue svariate opere. Un'analisi delle informazioni sugli esemplari delle sue opere in edizioni italiane conservati nelle biblioteche catalane e ricavabili dal catalogo collettivo consultabile in rete (CCUC: http://ccuc.cbuc. cat $/{ }^{*}$ cat) ci consente di osservare innanzitutto due fenomeni. ${ }^{9}$ Il primo consiste nella notevole varietà di opere conservate. Infatti, sebbene la Storia sia presente in un numero di esemplari e di edizioni proporzionalmente più alto (12 esemplari su 29, vale a dire circa il 40\%), si registrano edizioni antiche, cioè anteriori alla guerra civile, di molti altri testi desanctisiani (dalle due serie di Saggi critici alle lezioni su Mazzini e su Manzoni o ai volumi della serie La letteratura italiana nel secolo XIX, dal Saggio su Petrarca a quelli su Leopardi, da un'edizione scolastica di Prose scelte alle Pagine dantesche, dalle Lettere a Virginia a La giovinezza). Il secondo fenomeno riguarda invece la cronologia di questi testimoni, che registra un andamento non omogeneo nel tempo e sbilanciato, in positivo o in negativo, verso alcuni periodi. Spicca, su un versante, l'assenza di edizioni ottocentesche, il che pur non escludendo che fossero circolate, è indizio comunque che tale circolazione fosse scarsa. Sul polo opposto, diventa significativa, per contrasto, l'impennata che si registra nel primo dopoguerra, in coincidenza, cioè, con un periodo di particolare espansione dell'italianismo catalano avvenuta in seno al Noucentisme, impennata seguita poi da un progressivo diradarsi dei testimoni negli anni trenta.

7. El hombre de Guicciardini, in F. GuICCIARDINI, Recuerdos, trad. di Antonio Hermosa Andújar, Madrid: Centro de Estudios Constitucionales, 1988.

8. Cfr. Jaume Medina, La crítica literària i la història de la literatura, in J. Molas (dir.), Història de la literatura catalana, X, Barcelona: Ariel, 1987, p. 192.

9. L'inventario di volumi ricavato dal CCUC è riportato in appendice. 
Guardando più da vicino i dati, osserviamo poi come, dopo il testimone più antico, del 1907, cioè la prima edizione curata da Croce del Saggio sul Petrarca, nel corso del secondo decennio del secolo si registra una prima fase di diffusione segnata da una relativa continuità. Essa comincia nel biennio 1912-1913 grazie a due edizioni importanti per la fortuna italiana e internazionale di De Sanctis: la prima edizione laterziana della Storia curata da Croce nel 1912 (presente sia nella Biblioteca de Catalunya, sia soprattutto nella Biblioteca dell'Ateneu Barcelonès, centro nevralgico dell'intellettualità nella capitale) e la prima edizione commentata della Storia, dello stesso anno, la cosiddetta "prima edizione milanese» allestita da Paolo Arcari per il Treves di Milano (della quale si conserva la ristampa del 1913 nella Biblioteca dell'Universitat de Barcelona). ${ }^{10}$ Poi, a cavallo del centenario dantesco si concentra il momento di maggior circolazione di libri desanctisiani, anzi il 1921 è l'anno in cui datano il più alto numero di esemplari italiani conservati, cioè 5 , ed è anche l'anno della traduzione di Capdevila. Nel terzo decennio si rileva ancora una certa quantità di edizioni, mentre dopo il 1930 esse diventano sporadiche. La varietà delle opere, comunque, è costante in tutto il periodo considerato.

Ovviamente, si tratta di dati parziali e puramente orientativi, i quali d'altronde non possono che essere accresciuti dallo spoglio di altre biblioteche private o pubbliche. Di fatti, se proviamo a rileggere tali dati alla luce di un altro fondo interessante e nutrito, quello dei libri desanctisiani appartenuti al suo primo traduttore, Josep Maria Capdevila, ${ }^{11}$ il risultato conferma in sostanza il quadro precedente, salvo una novità rilevante, cioè la comparsa di due edizioni ottocentesche (i Nuovi saggi critici del 1879 e gli Scritti critici nell'edizione postillata da Imbriani), edizioni che presumibilmente Capdevila, nato nel 1892, aveva ereditato dalla biblioteca familiare.

Infine, un'altra informazione che non va trascurata è quella riguardante gli antichi proprietari di alcuni degli esemplari conservati. In particolare, l'edizione del 1907 del Saggio sul Petrarca fa parte del legato di Joan Vidal Alcover e di M. Aurèlia Capmany (e quindi anche in questo caso si tratterebbe di un'eredità familiare di uno dei due scrittori). Un'edizione Treves del 1921 della Storia è quella che possedeva Josep Pla, mentre una del 1922 per i tipi di Morano proviene dal fondo appartenuto a Joan Crexells. Molto più corposo è l'apporto della biblioteca di Carles Riba lasciata in donazione alla Biblioteca de Catalunya con le sue otto opere desanctisiane, una varietà comparabile a quella dell'altrettanto nutrito fondo posseduto da Capdevila.

10. Quanto all'importanza di Croce nella diffusione di De Sanctis, si tenga conto che nella Biblioteca de Catalunya si conservano anche due esemplari del primo importante saggio bibliografico da lui pubblicato nel 1917, Gli scritti di Francesco De Sanctis e la loro varia fortuna.

11. Il Fondo Capdevila appartiene attualmente alla Biblioteca del Seminario Diocesano di Girona. È possibile consultarne il catalogo alla pagina: http://www.seminaridegirona.com/ docs/fonscapdevila.pdf. 
Tutto quanto detto finora potrebbe già bastare a smentire il carattere drastico della dichiarazione di Josep Pla citata in apertura e a prospettare l'ipotesi di una diffusione delle opere e del pensiero di De Sanctis situabile nel secondo e terzo decennio del Novecento con un picco intorno al 1920-1922, in coincidenza con i fermenti italianisti che precedono e seguono il centenario dantesco. Senonché, è lo stesso Pla, in realtà, a fornirci altrove indizi in questa direzione, in particolare nel Quadern gris dove racconta di quando e come cominciò a interessarsi per la cultura italiana:

Vaig començar a estudiar l'italià per llegir la «Història de la literatura italiana» de Francesco de Sanctis, llibre d'un interès decisiu — segons l'opinió de Josep M. ${ }^{a}$ Capdevila i del seu inseparable Joan Climent. Josep M. ${ }^{a}$ Capdevila semblava tenir llavors tres obsessions: sant Francesc de Sales, Francesco de Sanctis i Josep Joubert. Climent el seguia. Aquestes tres obsessions havien estat creades per Eugeni d'Ors, naturalment. [...] $]^{12}$

Di qui che si iscrive a un corso di lingua italiana alla Casa degli Italiani, ${ }^{13} \mathrm{e}$

[p]ocs dies després d'haver començat el curs, demano a la biblioteca de l'Ateneu la Història de De Sanctis. No arribo a confegir res. Llegeixo un paràgraf $\mathrm{i}$ a la fi és com si no hagués llegit res. Faig la mateixa provatura amb un volum de la "Crítica» de Croce i arribo al mateix resultat. ${ }^{14}$

A questo punto, continua lo scrittore, si prefigge di trasferirsi per qualche tempo in Italia, dove, come ben noto, sbarcherà nel 1922. L'episodio è raccontato nell'annotazione dell' 8 settembre del 1919, ma come se fosse anteriore. Pla non ne precisa l'anno, lo fa risalire al periodo dei suoi studi universitari, cioè fra il 14 e il 19, di fatto è situabile non prima del gennaio 1918, quando Pla diventa socio dell'Ateneu, e non oltre l'aprile del 1920, quando parte per Parigi come corrispondente de La Publicidad. ${ }^{15}$ Intanto, l'aneddoto ci rivela almeno un paio di cose: anzitutto, che era stato l'ambiente noucentista a spingerlo verso la lettura di De Sanctis, ${ }^{16}$ e poi, che aveva trovato all'occorrenza anche un esemplare della Storia disponibile in biblioteca. Ma quel che

12. J. PlA, El quadern gris, Barcelona: Destino, 1983, vol. 2, p. 276.

13. Il professore che teneva il corso, che Pla ribattezza con il nome di "Cavaradosi», era Benedetto Colarossi (cfr. Silvio SANTAGATI, La Casa degli Italiani. Storia della comunità italiana di Barcellona (1865-1936), Barcelona: Editorial Mediterrània, 2007, p. 259-260).

14. J. PlA, El quadern gris, op. cit., p. 277.

15. Come riporta Xavier Febrés (Josep Pla: biografia de l'homenot, Barcelona: Plaza \& Janés, 1990, p. 37), Pla vi si iscrive il 5 gennaio 1918 come «soci transeünt». Nel diario manoscritto del 1918-1919, Pla annota in data 12 gennaio 1919: «Em dono d'alta a l'Ateneu» (cfr. l'edizione facsimile El primer Quadern gris, a cura di Xavier Pla, Barcelona: Destino, 2004). In ogni caso, nel manoscritto, che si interrompe nell'estate del 1919, non compare alcun riferimento né al corso d'italiano né a De Sanctis.

16. Nel volume Itàlia i el Mediterrani, Pla rifonde in parte e con varianti la storia della sua prima scoperta dell'Italia e dell'italiano esposta nel Quadern gris. In particolare, vi ricollega la lettura di De Sanctis all'influenza di Capdevila: «Potser, a Barcelona, Josep Maria Capdevila em recomanà, amb gran insistència, la "Storia della letteratura italiana” de De Sanctis» (op. cit., p. 402). 
succede a Pla, succede di pari passo anche ad altri, perché la scoperta di De Sanctis e la sua assunzione a modello critico diventa un fenomeno collettivo e generazionale negli anni che seguirono la prima guerra mondiale. Durante la guerra, la francofilia politica e ideologica aveva implicato una francofilia culturale e letteraria dominante e incontrastata negli ambienti intellettuali, la quale in certa misura aveva contrarrestato l'italianismo promosso già da prima dal Noucentisme. ${ }^{17}$ Il nuovo scenario storico favorisce, invece, la ripresa e la propaganda di quel discorso italianista, creando condizioni propizie anche alla diffusione di De Sanctis. È illuminante in questo senso quanto racconta in un articolo di giornale del 1927 lo scrittore di formazione modernista Puig i Ferreter riportando la testimonianza di un amico: ${ }^{18}$

- Uns anys enrera —em diu— ningú de nosaltres, els de la meva generació ni els de la generació que ens precedí, manifestà conèixer de Sanctis. Jo confesso que no en sabia res. La primera referència d'ell la vaig tenir per un escriptor francès que en feia un gran elogi [...]

—Això — afegeix l'amic - desvetllà la meva curiositat i vaig fer-me trametre les obres del crític italià. D'antuvi m'arribaren tres volums de «Saggi critici», que vaig devorar en pocs dies. Però la «Història», la gran «Història de la literatura italiana» no acabava de venir mai. Figura't la meva sorpresa quan un capvespre la vaig veure exposada a l'aparador d'una de les nostres llibreries estrangeres. Jo la tenia encarregada a una altra casa. Trobant-se aquesta obra a Barcelona, jo no em resignava a no llegir-la aquella mateixa nit. No portava diners, cosa que em passa molt sovint, i vaig córrer a manllevar-ne a un amic, el qual m'acompanyà fins a la mateixa llibreria tot estranyant el meu interès apassionat per aquella obra d'erudició [...].

—Entusiasmat amb aquest gran italià — diu el meu amic — no vaig tenir repòs fins que em vaig procurar l'estudi sobre Leopardi, les lliçons d'història de la literatura italiana del segle dinovè, recollides i prologades per Croce, i tot el que vaig poder de l’obra desanctisiana. [...] ¿Però no és ben curiós el que succeí llavors? Mentre feia aquestes lectures, vet ací que apareix a «La Veu de Catalunya» un article de Jordi March sobre de Sanctis, lucidíssim i sòlid com tot el que ell fa. Poc temps després una casa castellana editava els estudis de de Sanctis sobre la «Divina Comèdia». I a no trigar, Josep Maria Capdevila parlava de l'escriptor italià a la mateixa "Veu» i publicava en català i comentava els estudis esmentats.

17. È interessante al riguardo ciò che racconta lo stesso Pla nella pagina del Quadern gris già citata dell'8 settembre del 1919, p. 276: «Jo havia viscut fins a la data en un ambient francòfil. La penya de l'Ateneu, que havia tingut un paper tan important en el curs de l'última guerra, era d'una francofília delirant i frenètica. França i res més! [...] Tot el que feia referència a Itàlia tenia un cert descrèdit, una consideració molt petita. Eugeni d'Ors havia parlat d'Itàlia, a la penya, molt seriosament. [...] Les primeres persones del país que havien anat a Itàlia fora de les peregrinacions a Roma havien estat els noucentistes. [...] Pero, en fi, tot aquest moviment havia estat més aviat apàtic i s'havia mantingut en un terreny minoritari». Sull'italianismo del primo dopoguerra, cfr. Gabriella GAVAGNIN, Classicisme i Renaixement: una idea d'Itàlia durant el Noucentisme, Barcelona: Publicacions de l'Abadia de Montserrat, 2005.

18. «De Sanctis i Catalunya», La Publicitat, 8-X-1927. 
La circoscrizione temporale (tra il 1919 e il 1925, stando alle pubblicazioni cui si allude) di questo fenomeno di ricezione, che appare alla sensibilità dei contemporanei tanto improvviso quanto di ampia risonanza, coincide in sostanza con le date che abbiamo dedotto dalle edizioni censite nei fondi bibliotecari. In questi anni, quindi, si può parlare di una propagazione dell'interesse e della conoscenza di De Sanctis fuori dalla stretta cerchia dei singoli sostenitori, i quali avevano avuto modo, già dagli anni anteriori alla guerra, di avvicinarsi alle sue opere, per diretta influenza di Ors, come sostiene Pla, o no. Intanto, Puig i Ferreter, prima di concludere l'articolo con un appello a proseguire sulla stessa strada, ${ }^{19}$ segnala anche, in Carles Riba e Josep M. Capdevila, i due esempi più significativi di questa benefica influenza del pensiero di De Sanctis.

Effettivamente, di questa ricezione catalana primonovecentesca, Riba e Capdevila vanno considerati a ragione i due massimi esponenti, e non solo per lo sforzo di divulgazione svolto con elzeviri e traduzioni, ${ }^{20}$ ma soprattutto perché seppero trasformare la lezione desanctisiana in un modello critico operativo, applicandone principi, suggerimenti, categorie e perfino il linguaggio alla critica che esercitarono sulla letteratura catalana. Ciascuno dal proprio orizzonte ideologico e letterario, utilizzarono De Sanctis non solo come una chiave di lettura della storia letteraria italiana, di autori come Dante, Petrarca, Ariosto o Leopardi, o di epoche storiche come il Rinascimento, come altrove ho già cercato di dimostrare, ${ }^{21}$ ma anche e soprattutto come un metodo solido, duttile e nuovo per fare critica letteraria.

Per quanto riguarda Capdevila, meriterebbe senza dubbio un'analisi più approfondita di quella che mi è consentita di fare in questa sede. ${ }^{22} \mathrm{La}$ sua

19. «Benvingut sigui de Sanctis a Catalunya. Ell hi ha penetrat per camins diversos, hi ha trobat un terreny favorable. No ens allunyem massa aviat d'ell, que la seva influència no sigui fugissera. Impulsem el seu estudi. Ens pot ensenyar moltes coses bones, i no solament els crítics, ans més aviat als creadors, i la principal de totes és la fusió harmoniosa de l'art i de la vida: la vida com a contingut i l'art com a contingent, que fou el seu credo literari, el que predicà i practicà fins a la mort».

20. Vanno segnalati in primo luogo gli interventi cui si riferisce Puig i Ferreter, cioè l'escoli che Riba dedicò a De Sanctis (Jordi MarCH, «De Sanctis», La Veu de Catalunya, 8-II-1919, poi pubblicato in volume in Escolis $i$ altres articles, Barcelona: Publicacions de "La Revista», 1921) e gli articoli di Capdevila: «Francesco De Sanctis», La Publicitat, 5-II-1921 e «Una pàgina de Francesc de Sanctis. L'individualisme», La Veu de Catalunya, 14-II-1922, poi raccolti nel volume Poetes $i$ crítics, Barcelona, Llibreria Catalònia, 1925. Ma di Capdevila vanno ricordati anche le pagine che commentano, traducendone ampi stralci, $i$ saggi su Guicciardini (L'uomo de Guicciardini, anch'esso incluso in Poetes i crítics, op. cit.) e su Manzoni («Manzoni», La Paraula Cristiana, n. 36, dicembre 2007, p. 484-498).

21. Cfr. G. Gavagnin, Classicisme i Renaixement..., op. cit.

22. L'infuenza di De Sanctis su Capdevila è stata ribadita più volte dallo stesso autore, e ricordata, oltre che da Josep Pla, dai critici che si sono occupati di lui (MoLAS, Josep M. Capdevila, critic literari (1966), in Lectures critiques, Barcelona: Edicions 62, 1975, p. 83-89 e 
conoscenza di De Sanctis risale a prima della guerra: nel 1914, come attestato dalla corrispondenza, fa arrivare lo Studio su Giacomo Leopardi al mentore e amico Josep M. de Garganta. Capdevila era allora ventiduenne ma, grazie all'incoraggiamento di Garganta, membro dell'Arcadia italiana, aveva già una formazione italianista perché aveva cominciato a leggere scrittori italiani classici e moderni all'età di 16 anni. ${ }^{23}$ Tempo a venire, nel 1926, pubblicherà la sua prima raccolta di saggi, Poetes $i$ crítics, un libro rilevante nell'orizzonte critico dell'epoca. Qui la presenza di De Sanctis è costante, spesso è direttamente chiamato in causa, il che favorisce peraltro una certa predilezione per un approccio comparativo fra autori catalani e italiani: Alcover-Leopardi, Verdaguer-Tasso, Costa-Parini, etc. Ma anche quando il suo nome non appare citato esplicitamente avvertiamo nozioni, valori, dicotomie e procedimenti a lui riconducibili: dall'opposizione fra poeta e artista alla ricerca della passione e della vitalità come motori del testo, dalla gestione del rapporto fra mondo interno ed esterno al poeta alla ricerca del fondo umano depositato nell'opera. Vediamone qualche esempio.

La poesia di Josep Carner è valorata in quanto armonico risultato di un perfetto accordo fra il poeta e l'artista:

I llavors quan el poeta parla de la passió més profunda, la mà de l'artista és més ferma, el vers no es deslliga, no es desfá; l'artista el domina i marca les simetries de compàs, les concordàncies d'imatges, de conceptes. ${ }^{24}$

$\mathrm{Su}$ Costa, Capdevila soppesa positivamente il riflesso della personalità dell'autore nell'opera:

Però l'home domina en la seva obra; l'home moral que hi havia en el poeta mostra, a cada descripció, la seva vigoria interna. ${ }^{25}$

Ancora, a proposito dello stile di Costa i Llobera, da un lato sanziona l'eccessivo controllo esercitato sul linguaggio, che denota assenza di appassionamento:

És un estil culte com el de les poesies de Manzoni o de Parini. Desconfia del seu instint, i per aqueixa desconfiança, contenint l'instint verbal, en ell meravellós, cau de vegades en la fredor i trenca les ales del vers. ${ }^{26}$

M. Serrahima, Josep Maria Capdevila. Assaig biogràfic, Barcelona: Barcino, 1974). Nuovi dati sono stati apportati dallo studio di Joan CARRERAS I Pera, Josep Maria Capdevila. Ideari i poètica, Barcelona: Publicacions de l'Abadia de Montserrat, 2003, p. 144-148.

23. L'epistolario di Josep Maria Capdevila a Josep Maria de Garganta è stato pubblicato in appendice alla Tesi di Dottorato di Joan Carreras I Pera, Ideari i poètica de Josep Maria Capdevila, Universitat Autònoma de Barcelona, 2001, ed è ora consultabile in rete: http:// www.tesisenxarxa.net/TESIS_UAB/AVAILABLE/TDX-1004102-144116//jcp6de6.pdf

24. Poetes i crítics, op. cit., p. 149.

25. Ibid., p. 53.

26. Ibid., p. 45. 
dall'altro, però, riconosce nei suoi versi una sempre sincera dialettica tra forma e contenuto al riparo da ogni uso artificioso e vuoto, secondo un metro di giudizio applicato tante volte da De Sanctis: ${ }^{27}$

era incapaç d'artifici, en el sentit de simular un fons inexistent o de consagrar a capricis de forma estrofes buides. ${ }^{28}$

Quanto al rapporto mondo interno-esterno al poeta, valga questa riflessione su Carner:

sempre imposa la selecció seva al món de fora, i solament quan es parla ell sol minva aquelles reserves. ${ }^{29}$

Ma anche lo stile di Capdevila riesce ad essere di stampo desanctisiano. Si legga, a proposito di Verdaguer:

Però el geni, si el cerquem, el trobem; si no el cerquem, tot d'una se'ns imposa. ${ }^{30}$

Se dovessi, però, indicare in sintesi che cosa Capdevila ammira di più in De Sanctis, direi che è soprattutto la lezione morale e pedagogica che informa i suoi scritti. "Mai el crític ni l'erudit — scrive — van a part de l'home d'acció, de l'educador, del polític. A propòsit de l'anàlisi literari d'una obra, entra dins l'esperit d'ella i llavors hi aplica la seva mesura, el seu cànon, i acaba parlant l'educador i el polític». ${ }^{31}$ E Capdevila, che come segnala Maurici Serrahima, possedeva una «innegable i poderosíssima vocació pedagògica», ${ }^{32}$ si adoperò per divulgare, da tale ottica, l'insegnamento di De Sanctis.

Il peso specifico che ha De Sanctis nella formazione della poetica e nell'esercizio della critica ribiana è stato già richiamato da tempo dalla critica. ${ }^{33}$ Più di recente, le monografie di Jordi Malé hanno contribuito a esplicitare con riscontri testuali i debiti ribiani nei confronti del critico irpino. ${ }^{34}$ Non potendo addentrarmi in modo sistematico nell'argomento, preferisco soffermarmi su un paio di aspetti, perché a mio avviso singolarizzano la lettura quanto mai minuziosa che Riba fa di De Sanctis. Una delle questioni di metodo che Riba coglie nei suoi scritti è l'attenzione rivolta alla struttura

27. Si veda per esempio quest'affermazione nel Saggio sul Petrarca: "Questa bella forma non è puro artificio tecnico, una costruzione meccanica fatta a freddo ed a priori; ma è il prodotto della sua anima».

28. Ibid., p. 43.

29. Ibid., p. 155 .

30. Ibid., p. 39.

31. J.M. CAPDEVILA, «Francesco De Sanctis», art. cit.

32. M. Serrahima, op. cit., p. 102.

33. Cfr. Giuseppe E. SAnsone, L'obra crítica de Carles Riba, dins C. Riba, Obres Completes/2, Crítica, 1, a cura d'E. Sullà, Barcelona: Edicions 62, 1985 [1967'].

34. Cfr. Carles Riba i el Noucentisme. Les idees literàries (1913-1920), Barcelona: Edicions de la Magrana, 1995 e Poètica de Carles Riba (1920-1938), Barcelona: Edicions de la Magrana, 2001. 
interna del testo. Per esempio, De Sanctis descrive come un valore la forza con cui un autore riesce ad aggregare intorno a un centro vitale gli altri elementi testuali mentre, al contrario, connota come un disvalore, l'assenza di tale solidarietà interna. Nel primo caso, il concetto è esemplificato a proposito della Commedia:

La creazione comincia veramente, quando quel mondo tumultuario e frammentario trovi un centro intorno a cui stringersi. Allora esce dall'illimitato che lo rende fluttuante, e prende una forma stabile; allora nasce e vive, cioè si sviluppa gradatamente secondo la sua essenza. Ora il mondo dantesco trovò la sua base nella idea morale. ${ }^{35}$

\section{Nel secondo, a proposito della canzone Perché la vita è breve di Petrarca:}

La materia che vuol trattare non è ancora organizzata; le idee gli stano innanzi senza colore e senza calore; e, come non hanno trovato un centro intorno a cui raggrupparsi e comporsi, fluttuano nello spazio a guisa di atomi erranti, che vorrebbero unirsi e non hanno ancora la forza d'attrarsi. ${ }^{36}$

Questa riflessione, strutturalista avant la lettre, è ripresa da Riba in varie occasioni ed è poi rielaborata nella misura in cui diventa una preoccupazione centrale nella sua poetica. Nella recensione a La vida nua di Lluís Valeri, l'idea è applicata da Riba a partire da una citazione esplicita dalla Storia,${ }^{37}$ in cui De Sanctis, a proposito dell'Inferno, scriveva che

la passione raccoglie tutte le forze interiori, distratte e sparpagliate nell'uso quotidiano della vita, intorno a un punto solo, di modo che lo spirito acquista la coscienza della sua libertà infinita. ${ }^{38}$

$\mathrm{Ma}$ in un altro articolo ribiano di un anno e mezzo prima, dedicato a La nau di Ventura Gassol, pur non essendovi alluso De Sanctis, il concetto già appariva con riprese letterali di questo stesso stesso passo:

La passió del nostre Ventura Gassol, en canvi, sembla necessitar, com el vent, un espai sense límits. No és tensió, ni tan sols arremorament de forces entorn d'un sol punt del sentiment o de la intel.ligència; sinó més aviat el voler que s'amolla en una avinentesa d'infinita llibertat. ${ }^{39}$

In una prospettiva analoga, Riba fa appello a un altro principio strutturante che preoccupa De Sanctis, anzi a uno dei principi metodologici cardinali nella sua prassi critica: la sintesi unitaria fra ideale e reale, fra universale e particolare così come essa viene realizzata in determinate opere letterarie. L'esem-

35. Storia della letteratura italiana, Torino: Einaudi, 1981, I, p. 188-189.

36. F. De SANCTIS, Saggio critico sul Petrarca, Torino: Einaudi, 1952, p. 145-146.

37. C. RibA, Obres completes / 2. Crítica, 1, op. cit., 1985, p. 256. La recensione risale al 17 dicembre 1921.

38. Storia della letteratura italiana, op. cit., 209.

39. Ibid., p. 190-191. Il passo è commentato da Jordi Malé, Carles Riba i..., op. cit., p. 266. 
pio che citerò proviene da un articolo ricco di un'intertestualità implicita con i testi desanctisiani e dedicato alla poesia di López-Picó. ${ }^{40}$ Questa, scrive Riba, a differenza di quanto succede in Leopardi, il Leopardi degli idilli, non partirebbe da una convenzione generale ma da un motivo personale, anzi, intimo. ${ }^{41}$ Il problema, dunque, il limite, anzi, è che nella poesia di López-Picó, "[l]'objecte poètic li roman, doncs, darrera un nom com darrera una paret: la imaginació no pot fondre's amb ell, generant la nova realitat artística, total, simple $i$ clara [corsivi miei]». ${ }^{42}$ Tanto la specifica lettura leopardiana ${ }^{43}$ quanto la recriminazione a López-Picó di non esser riuscito a "fondere» immaginazione e oggetto poetico si ispirano a un passaggio di De Sanctis su A Silvia (ora nello Studio su Giacomo Leopardi), da cui proviene, oltre alla ripresa della serie aggettivale - chiara semplice totale —, soprattutto il concetto di fusione come azione che permette il passaggio dall'io al genere umano, senza che si perda il carattere reale e non astratto dell'esperienza vissuta.

$\mathrm{Ci}$ è qui dunque tutta quella partecipazione d'anima che è necessaria per dare a questa storia un carattere d'individualità, quel finito, quel contorno ch'è proprio di cosa reale.

La situazione è così chiara come semplice, la formazione è totale.

Ma questa poesia oltrepassa il limite di un fatto individuale e prende proporzioni colossali. [...] Questo è carattere comune a tutta la poesia leopardiana; il senso generale fa stacco a modo di sentenza o di riflessione, e talora raffredda l'impressione che ti viene dal particolare. Ma qui è fusione completa. Il senso umano della poesia è così intimamente fuso col senso individuale che

40. Il testo è pubblicato nel maggio del 1921, poco dopo che l'amico Josep Obiols, ormai ritornato a Barcellona, gli aveva fatto arrivare i libri che Riba gli aveva incaricato di comprargli in Italia: «I les obres de De Sanctis que jo no tingui, i que Vós conegueu que tenen un interès literari. Jo tinc: La Storia de la Lett ${ }^{\mathrm{a}}$ Italiana; els Saggi critici. - Em manquen, doncs el Saggio sobre el Petrarca i el sobre Leopardi. No sé —no tinc aquí la llista - si hi ha uns altres reculls de Saggi critici. Per guiar-vos, els que jo tinc són 4 volumets petits, i comencen amb uns estudis sobre el Dant, entre ells un sobre Beatriu» (Lettera a Obiols del 14 febbraio 1921, in Cartes de Carles Riba. I: 1910-1938, a cura de Carles-Jordi Guardiola, Barcelona: Edicions de la Magrana, p. 134). I titoli qui elencati sono tutti presenti nel fondo della Biblioteca de Catalunya proveniente dalla Donazione (cfr. il catalogo in appendice).

41. Il richiamo a Leopardi non è gratuito ma stimolato dal fatto che nei versi di López-Picó appare un motivo riconducibile alla rete tematica leopardiana, cioè «el deseiximent melangiós de l'endemà de la festa»: "Leopardi cantà el dissabte il.lusionat i el melangiós vespre de diumenge, elevant-los a al-legories de la fugacitat del goig i de la persistència de l'afany. Però no medità sobre un nom, sinó sobre un idil.li humà, concret i genèric alhora, que donava fesomia al dia. / En el seu Septenari, J. M. López-Picó sembla centrar la sèrie en una anècdota diumengera d'amor. És a dir, no parteix d'una convenció general, com Leopardi, sinó d'un motiu personal, i més encara, íntim» (C. RiBA, Obres completes / 2. Crítica, 1, op. cit., p. 235).

42. Ibid., p. 236.

43. De Sanctis sottolinea questo passaggio dal personale all'universale nel saggio Gli idilli, concretamente a commento dei versi 30-33 della Sera del dì di festa, cfr. F. De SANCTIS, La letteratura italiana nel secolo decimonono. XIII, Leopardi, a cura di C. Muscetta e Antonia Perna, Torino: Einaudi, 1969, p. 120-121. 
sembra parte di quello e che venga fuori come eccitato e provocato nel vivo della passione, quasi un crescendo. ${ }^{44}$

Queste riflessioni sul funzionamento del testo di cui Riba faceva tesoro e rielaborava nella sua prassi critica si coniugavano perfettamente con la sua ricerca teorica maturata in un orizzonte poetico postsimbolista, gli fornivano degli strumenti che poteva proficuamente integrare con quelli ricavati da Vossler o Valéry.

Ben diverso è il De Sanctis di Josep Pla, che è fondamentalmente il De Sanctis della Storia, libro che Pla legge e rilegge in vari momenti nel corso della sua vita, contribuendo poi a divulgare fra i lettori catalani nella seconda metà del Novecento mediante ripetuti riferimenti in testi come el Quadern gris (1966) o La vida amarga (1967) e soprattutto mediante la traduzione dei capitoli su Machiavelli e su Aretino in Itàlia i el Mediterrani. ${ }^{45}$ Giova ricordare, peraltro, che la sua funzione di mediatore è potenziata dalla sua popolarità in quanto giornalista e scrittore. Pla usa la Storia della letteratura italiana come una ricca fonte di informazione sull'Italia, quasi una Baedecker culturale, ${ }^{46}$ la definisce addirittura «llibre bàsic per a conèixer més o menys aquest país». ${ }^{47}$ Non solo. Pla vi scorge anche qualcos'altro, fondamentalmente due cose. Da un lato, un modo di parlare della storia, della cultura, della letteratura e della società attraverso la costruzione di ritratti umani, di personalità complesse, esplorate nella loro vita materiale e morale, penetrate nella loro essenza, raccontate nella loro quotidianità. D'altronde, lo scrittore degli homenots non poteva non ammirare i ritratti di queste «enormes figures» che per Pla condensano l'essenza di un paese. Dall'altro, il principio secondo cui lo scrittore ha una responsabilità storica, e pertanto la letteratura deve fare i conti con la realtà in cui viene prodotta. Sulla base della Storia di De Sanctis, Pla giustifica la sua difesa del realismo, della letteratura interpretata con militanza in chiave antiidealistica. È noto il passo in cui Pla manifesta tale principio poggiando sull'autorità desanctisiana:

La primera obligació d'un escriptor és observar, relatar, manifestar l'època en què es troba [...]. La literatura és el reflex d'una societat determinada en

44. Ibid., p. 370-371. Occorre dire il testo Silvia non venne incluso nello Studio su Leopardi fino all'edizione di Nino Cortese del 1933, ma in quegli anni Riba avrebbe potuto leggerlo nel volume curato da Croce Scritti varii inediti e rari (1898).

45. Non ho trovato alcuna allusione a De Sanctis nelle opere di Pla pubblicate prima della guerra, nemmeno in un libro come Cartes meridionals (1929), dove pure spesseggiano rinvii alle più svariate fonti bibliografiche sulla cultura italiana, da Sthendal a Goethe, da Burckhardt a Gebhart, da Vasari a Papini. Di contro, è significativa la presenza costante di De Sanctis nel volume Itàlia i el Mediterrani, al margine ovviamente delle parti tradotte. I primi cenni a De Sanctis compaiono proprio nei testi degli anni sessanta sopra citati. È lo stesso Pla a ragguagliarci ancora una volta sulla cronologia delle sue letture; nelle Notes per a un diari gener 1967 - octubre 1968, scrive in data 16 maggio 1967: "A la matinada, llegida la "Història de la literatura italiana" de De Sanctis en relació amb el Bembo" (El viatge s'acaba, Barcelona: Destino, 1981, p. 42).

46. J. Pla, Itàlia i el Mediterrani, op. cit., p. 312.

47. Ibid., p. 316. 
un determinat moment. L'axioma — vàlid des del temps més reculats— és de De Sanctis, i jo modestament el comparteixo. ${ }^{48}$

Dal canto suo, anche Riba si poneva la questione del rapporto fra letteratura e realtà, ma l'affrontava in modo un po' più problematico, in quanto gli risultava improponibile tradurre tale rapporto in un mero e immediato riflesso di questa in quella. Non stupisce, quindi, che, leggendo De Sanctis si soffermasse su una formulazione che precisava e relativizzava la funzione di mediatore del poeta e che verosimilmente appare più conciliabile con le sue aspirazioni poetiche. Il passo è evidenziato a matita nella copia a lui appartenuta del Saggio critico sul Petrarca e inoltre viene richiamato con l'espressione «el poeta i la realitat" in un succinto indice tematico, dotato anche delle pagine di riferimento, redatto a mano da Riba alla fine del volume per uso personale:

Ciò che si dee domandare al poeta è che, calando nel reale, non vi stagni, non vi s'insozzi; che vi guardi inviolata la libertà dello spirito e il sentimento dell'arte. $^{49}$

Certo si capisce perché Riba prendeva nota proprio di questa riflessione con cui De Sanctis individuava un principio importante del decalogo di un poeta. Riba vi trovava salvaguardata in questo modo la sostanziale indipendenza del mondo poetico nei confronti del mondo reale.

La ricchezza e la complessità del pensiero e del metodo critico di De Sanctis consentiva quindi a due scrittori come Pla e Riba, per tanti aspetti diversi, specie poi per le concezioni estetiche, di riuscire a scoprirvi ciascuno la lezione teorica e metodologica più adeguata alla propria ricerca e pratica letteraria. È in questa pluralità di approcci e di letture che risiede, credo, la forza e l'intensità dell'influenza di De Sanctis in Catalogna.

48. J. Pla, La vida amarga, Barcelona: Destino, 1967, p. 8

49. F. De Sanctis, Saggio critico sul Petrarca, Napoli: Morano, 1921, p. 182. L'indice tematico manoscritto è a p. 316 . 


\section{Catalogo delle edizioni italiane anteriori al 1950 di opere di De Sanctis conservate in biblioteche catalane}

\section{Opere conservate nelle biblioteche del catalogo digitale CCUC ${ }^{50}$}

1907

Saggio critico sul Petrarca di Francesco De Sanctis. Nuova edizione a cura di Benedetto Croce, Napoli: A. Morano. [URV: Legato Vidal-Capmany]

1912

Storia della letteratura italiana, a cura di Benedetto Croce, Bari: Gius. Laterza \& Figli, 2 vol. [BC]; [BAB]

\section{3}

Storia della letteratura italiana, nuova edizione riveduta e corretta, Napoli: Alberto Morano, 2 vol. [RABL]

Storia della letteratura italiana, Milano: Fratelli Treves, 2 vol. [UB: l'esemplare era appartenuto alla biblioteca dell'Ateneu Enciclopèdic Popular]

\section{5}

La giovinezza di Francesco De Sanctis, frammento autobiografico pubblicato da Pasquale Villari, Napoli: A. Morano, 5a ed. [BC: Fondo August Matons]

\section{4-1916}

Saggi critici, a cura di Michele Scherillo, nuova edizione riordinata, accresciuta e corretta, Napoli: A. Morano, 4 vol. [BC: Donazione Riba-Arderiu. Sul primo dei 4 volumi è scritta a matita la firma di Carles Riba e la data d'acquisizione: «Perugia set 1920»]

1917

Lettere a Virginia, edite da B. Croce, Bari, Gius. Laterza \& Figli. [BC]

\section{9}

Nuovi saggi critici, Napoli: A. Morano. [BC: Donazione Riba i Arderiu. Contiene firma autografa di Carles Riba]

Studio su Giacomo Leopardi: opera postuma, curata dal prof. Raffaele Bonari, Napoli: A. Morano, 6a ed. [BC: Donazione Riba i Arderiu. Contiene firma autografa di Carles Riba]

1920

Prose scelte: per le persone cólte e per le scuole, a cura di Michele Scherillo, Napoli: Alberto Morano, $3^{\mathrm{a}}$ ed. [BC: Donazione Riba i Arderiu]

50. Il catalogo è stato consultato nel dicembre 2008. Si indica la biblioteca di appartenenza con le sigle usate nel catalogo. 
Scritti critici, con prefazione e postille di Vittorio Imbriani, Napoli: Alberto Morano, 6a ed. [BC: Donazione Riba i Arderiu. Contiene firma autografa di Carles Riba]

Storia della letteratura italiana, vol. 1, Milano: Fratelli Treves. [UdL]

1921

Pagine dantesche, con prefazione e note di Paolo Arcari, Milano: Fratelli Treves [BC]; [BPA]

Saggio critico sul Petrarca di Francesco De Sanctis, a cura di Benedetto Croce, Napoli: Alberto Morano, nuova ed. [BC: Donazione Riba i Arderiu. Contiene firma autografa di Carles Riba. Annotazioni a matita alla p. 316].

Storia della letteratura italiana, nuova edizione riveduta e corretta, Napoli: Alberto Morano, 2 vol. [BC]

Storia della letteratura italiana, prima edizione milanese a cura di Paolo Arcari, Milano: Fratelli Treves, 2 vol. [BJP]

1922

La letteratura italiana nel secolo XIX. Scuola liberale $=$ scuola democratica, lezioni raccolte da Francesco Torraca e pubblicate con prefazione e note da Benedetto Croce, Napoli: Alberto Morano, 7a ed. [BC: Donazione Riba i Arderiu] Manzoni: studi e lezioni, a cura di Giovanni Gentile, Bari:Gius. Laterza \& Figli. [BC]

Storia della letteratura italiana nuova edizione riveduta e corretta, Napoli: Alberto Morano, 2 vol., terza ristampa. [BC: Donazione Riba i Arderiu]; [BAB: Fondo Joan Crexells]

1925

Storia della letteratura italiana, Milano: Fratelli Treves, 2 vol. [RABL]

Storia della letteratura italiana, a cura di Benedetto Croce, Bari: Laterza, 2 vol. [UB]

1928

Mazzini: cinque lezioni, Bari: Gius. Laterza \& Figli, ristampa. [BC]

1930

Storia della letteratura italiana, a cura di Paolo Arcari, Milano: Fratelli Treves, 2 vol. [UAB]

1943

Un viaggio elettorale, a cura di Edmondo Cione, Milano: Bompiani. [UB]

1946

La giovinezza [frammento autobiografico], introduzione di Daniele Mattalia, Milano: Aldo Martello. [UB]

1950

La giovinezza [frammento autobiografico], a cura di Dario Puccini, Milano: Universale. [UB] 


\section{Opere conservate nel Fondo Josep Maria Capdevila della Biblioteca} del Seminari Diocesà de Girona

1879

Nuovi saggi critici, Napoli: A. Morano, 2a ed.

1895

Scritti critici di Francesco De Sanctis, con prefazione e postille di Vittorio Imbriani, Napoli: A. Morano, $5^{\mathrm{a}}$ ed.

1913

Saggio critico sul Petrarca di Francesco De Sanctis, nuova edizione a cura di Benedetto Croce, Napoli: Fratelli Morano.

Storia della letteratura italiana, nuova edizione riveduta e corretta, Napoli: Alberto Morano, 2 vol.

1914-1916

Saggi critici, a cura di Michele Scherillo, nuova edizione riordinata, accresciuta e corretta, Napoli: A. Morano, 4 vol.

Pagine dantesche, con prefazione e note di Paolo Arcari, Milano: Fratelli Treves.

1924

Saggi critici, a cura di Michele Scherillo, nuova edizione riordinata, accresciuta e corretta. Terza serie, Napoli: A. Morano.

$1930-1933$

La letteratura italiana del secolo decimonono, Napoli: Morano, 4 vol. (Opere complete a cura di Nino Cortese, vol. III-VI: III. Manzoni, 1931; IV. La scuola liberale, 1931; V. Mazzini e la scuola democratica, 1930; VI. Leopardi, 1933). 